\title{
Parameters Governing Invasive Disease Propensity of Non-M1 Serotype Group A Streptococci
}

\author{
Peter G. Maamary ${ }^{a, b}$ Martina L. Sanderson-Smith ${ }^{a, c}$ Ramy K. Aziz ${ }^{\text {d, e }}$ \\ Andrew Hollands ${ }^{a, g}$ Jason N. Cole ${ }^{a, g}$ Fiona C. McKay ${ }^{a}$ Jason D. McArthur ${ }^{a}$ \\ Joshua K. Kirk ${ }^{a}$ Amanda J. Cork ${ }^{a}$ Rachael J. Keefe ${ }^{a}$ Rita G. Kansal ${ }^{e}$ \\ Hongmin Sun ${ }^{i}$ William L. Taylor ${ }^{f}$ Gursharan S. Chhatwal ${ }^{c}$ David Ginsburg ${ }^{j}$ \\ Victor Nizet ${ }^{\mathrm{g}, \mathrm{h}}$ Malak Kotb ${ }^{\mathrm{e}, \mathrm{k}, \mathrm{l}}$ Mark J. Walker ${ }^{\mathrm{a}, \mathrm{b}}$
}

aSchool of Biological Sciences, University of Wollongong, Wollongong, N.S.W., and b School of Chemistry and Molecular Biosciences, University of Queensland, St. Lucia, Qld., Australia; 'Department of Microbial Pathogenesis and Vaccine Development, Helmholtz Centre for Infection Research, Braunschweig, Germany; ${ }^{\mathrm{d} D e p a r t m e n t}$ of Microbiology and Immunology, Faculty of Pharmacy, Cairo University, Cairo, Egypt; eVA Medical Center and ${ }^{f}$ Molecular Resource Center, University of Tennessee, Memphis, Tenn., 9 Department of Pediatrics and h ${ }^{\text {S }}$ kaggs School of Pharmacy and Pharmaceutical Sciences, UCSD, La Jolla, Calif., 'Department of Internal Medicine, University of Missouri, Columbia, Mo., ${ }^{j}$ Howard Hughes Medical Institute, Department of Internal Medicine and Department of Human Genetics, University of Michigan, Ann Arbor, Mich., ${ }^{k}$ VA Medical Center and 'Department of Molecular Genetics, Biochemistry and Microbiology, University of Cincinnati, Cincinnati, Ohio, USA

\section{Key Words}

Animal models $\cdot$ Bacteriology $\cdot$ Immunity $\cdot$ Innate $\cdot$

Neutrophils $\cdot$ Streptococcus $\cdot$ Virulence factors $\cdot$ Invasive infection

\begin{abstract}
Group A Streptococcus (GAS) causes rare but life-threatening syndromes of necrotizing fasciitis and toxic shock-like syndrome in humans. The GAS serotype M1T1 clone has globally disseminated, and mutations in the control of virulence regulatory sensor kinase (covRS) operon correlate with severe invasive disease. Here, a cohort of non-M1 GAS was screened to determine whether mutation in covRS triggers systemic dissemination in divergent M serotypes. A GAS disease model defining parameters governing invasive propen-
\end{abstract}

sity of differing $M$ types is proposed. The vast majority of GAS infection is benign. Nonetheless, many divergent $M$ types possess limited capacity to cause invasive infection. M1T1 GAS readily switch to a covRS mutant form that is neutrophil resistant and frequently associated with systemic infection. Whilst non-M1 GAS are shown in this study to less frequently accumulate covRS mutations in vivo, such mutants are isolated from invasive infections and exhibit neutrophil resistance and enhanced virulence. The reduced capacity of non-M1 GAS to switch to the hypervirulent covRS mutant form provides an explanation for the comparatively less frequent isolation of non-M1 serotypes from invasive human infections.

Copyright ๑ 2010 S. Karger AG, Basel

\section{KARGER}

(C) 2010 S. Karger AG, Basel

Fax +4161306 1234

E-Mail karger@karger.ch

www.karger.com
Prof. Mark J. Walker

School of Chemistry and Molecular Biosciences

The University of Queensland

Cooper Road, St. Lucia, Qld. 4072 (Australia)

Tel. +61 73346 1623, Fax +61 73365 4273, E-Mail mark.walker@uq.edu.au 


\section{Introduction}

Group A Streptococcus (GAS) causes 700 million infections each year, resulting in over 500,000 deaths. Invasive GAS infections account for $>600,000$ disease episodes, incurring a death rate of approximately $25 \%$ [1]. Over the past 30 years, a resurgence of life-threatening invasive GAS pathologies has been documented, in parallel with the emergence of the globally disseminated M1T1 clone $[2,3]$. The M1T1 clone remains the most frequently isolated M serotype from cases of invasive GAS infection and also from simple pharyngitis [4]. Nonetheless, other GAS serotypes cause invasive infections in Western populations, and GAS invasive disease in indigenous populations, such as Aboriginal communities of Northern Australia, is often associated with multiple M serotypes [5].

Historically, it is documented that hyperencapsulated GAS isolates are associated with invasive human infection and increased virulence in murine models [6-10]. Similarly, an inverse correlation has been described between SpeB production and disease severity in both human clinical disease and in murine models $[11,12]$. In the GAS M1T1 clone, mutations in the control of virulence regulatory sensor kinase ( $\operatorname{cov} R S$; alternatively designated csrRS) operon are selected for in vivo, and result in upregulation of capsule, loss of $\mathrm{SpeB}$ expression, increased disease severity in murine infection models and are more frequently associated with severe human invasive disease [10, 13-15].

The GAS M1T1 clone is distinguished from closely related M1 strains by the acquisition of the bacteriophageencoded DNase Sdal and superantigen SpeA $[16,17]$. Human neutrophil-mediated killing of GAS selects for the neutrophil-resistant covRS mutant form of M1T1. The acquisition of the bacteriophage-encoded $s d a 1$ gene provided M1T1 with enhanced capacity to switch to the covRS mutant form [14], as Sda1 mediates escape from neutrophil extracellular traps $[18,19]$. The loss of SpeB-mediated proteolytic degradation in vivo, as a result of $\operatorname{cov} R S$ mutation, preserves expression of Sda1 and other virulence factors [20], allowing GAS to recruit and activate the broad-spectrum human protease plasmin on the bacterial surface, resulting in extensive tissue destruction and triggering systemic dissemination [11, 14].

While significant advances in the understanding of GAS M1T1 invasive disease initiation have been made, parameters governing invasive propensity of other $M$ types have not been elucidated. Recently, analysis of GAS isolates of varying $\mathrm{M}$ type documented an association between invasive clinical isolates and mutations in genes encoding GAS global gene regulators (covRS/csrRS and ropB/rgg) [21]. In this study, we have examined a set of non-M1 serotype GAS isolates to determine whether such mutations trigger systemic dissemination in divergent $\mathrm{M}$ types. A model describing the invasive potential of differing $\mathrm{M}$ types is proposed.

\section{Materials and Methods}

GAS Strains and Culture Conditions

Clinical GAS isolates examined in this study have been described previously (table 1). Routine culture of GAS was conducted in stasis at $37^{\circ} \mathrm{C}$ in Todd-Hewitt broth supplemented with $1 \%$ (w/v) yeast extract or on horse-blood agar. GAS cultures for use in microarray experiments were propagated in Todd-Hewitt broth supplemented with $1.5 \%(\mathrm{w} / \mathrm{v})$ yeast extract.

SpeB Activity Assays

SpeB cysteine protease activity in cell-free stationary-phase supernatants was determined using the chromogenic substrate N-benzoyl-Pro-Phe-Arg- $p$-nitroanilide-hydrochloride (Sigma), according to the method of Hytönen et al. [22]. To screen large numbers of GAS colonies recovered following subcutaneous murine passage $(n=1,500)$, single colonies were transferred to designated grid locations on Columbia agar plates supplemented with $15 \%(\mathrm{v} / \mathrm{v})$ commercial skim milk (Devondale) and assayed for secreted SpeB activity as described by Ashbaugh et al. [23].

\section{Western Blot Analysis}

Stationary-phase supernatant proteins were concentrated 37.5 -fold in $100 \mathrm{mM}$ Tris ( $\mathrm{pH}$ 7.6) by precipitation with $10 \%$ trichloroacetic acid. SpeB protein was then detected using Western blot analysis essentially as previously described [11].

\section{Quantification of Hyaluronic Acid Capsule Biomass}

Overnight cultures in Todd-Hewitt broth supplemented with $1 \%(\mathrm{w} / \mathrm{v})$ yeast extract were subinoculated $1: 14$ and grown to midlogarithmic phase $\left(\mathrm{OD}_{600}\right.$ 0.6). Capsule extraction and quantification were conducted using the method of Ashbaugh and Wessels [24].

DNA Sequence Analysis of the covRS Locus

The method for mapping mutations in the GAS covRS operon was as described previously [14]. Genomic DNA was isolated using the QIAGEN DNeasy Blood and Tissue Kit (Qiagen) according to the manufacturer's guidelines. For each operon, sequences were assembled in Chromas Pro v1.33 (Technelysium Pty Ltd) and aligned with the intact covRS operon of the M1T1 isolate 5448 (BioEdit v7.0.9.0; Ibis Biosciences)

Microarray Design and Production

An oligonucleotide microarray with probes representing M1 core ORFeome in addition to ORFs representing various M1, M3, M18 and Streptococcus dysgalactiae prophages was used in this study. The microarray was an expansion of one described previously [25]. Oligomers (70mers) were obtained from Dr. Kevin McIver and Dr. June Scott, and printed in the Molecular Resource 
Table 1. Characteristics of GAS isolates and mutant strains utilized

\begin{tabular}{|c|c|c|c|c|}
\hline Isolate & emm type & Isolate origin & Clinical origin & Reference or source \\
\hline 5448 & 1.0 & USA & invasive; STSS/NF & {$[20]$} \\
\hline 5448AP & 1.0 & animal passage & generated during murine passage & {$[20]$} \\
\hline NS13 & 53 & Australia & invasive; blood & {$[37]$} \\
\hline NS88.2 & 98.1 & Australia & invasive; blood & {$[37]$} \\
\hline NS179 & 9.1 & Australia & invasive; blood; pustules on foot & {$[37]$} \\
\hline NS210 & 22 & Australia & invasive; diabetic ulcer with fever & [37] \\
\hline NS223 & 91 & Australia & invasive; blood & [37] \\
\hline NS452 & 25 & Australia & invasive; cellulitis; wound & {$[37]$} \\
\hline NS455 & 52 & Australia & invasive; blood & {$[37]$} \\
\hline NS501 & 14 & Australia & invasive; blood & {$[37]$} \\
\hline A 20 & 23 & Japan & invasive; blood & {$[41]$} \\
\hline NS730 & 90 & Australia & invasive; nf; pus from left hip & {$[37]$} \\
\hline NS733 & 90 & Australia & invasive; nf; wrist aspirate & [37] \\
\hline NS931 & 69 & Australia & invasive; nf; blood & {$[37]$} \\
\hline NS1133 & 101 & Australia & invasive; blood & [37] \\
\hline ALAB49 & 53 & USA & superficial; impetigo; skin lesion & {$[42]$} \\
\hline NS10 & 53 & Australia & superficial; throat swab & [37] \\
\hline NS14 & 102 & Australia & superficial; post-operative wound & {$[37]$} \\
\hline NS32 & 101 & Australia & superficial; wound infection & [37] \\
\hline NS50.1 & 108 & Australia & superficial; wound infection & {$[37]$} \\
\hline NS53 & 71 & Australia & superficial; fever & [37] \\
\hline NS59 & 53 & Australia & superficial; wound infection & {$[37]$} \\
\hline NS236 & 77 & Australia & superficial; sore throat; throat swab & [37] \\
\hline NS253 & 52 & Australia & superficial; wound infection & [37] \\
\hline NS265 & 56 & Australia & superficial; wound infection & [37] \\
\hline NS297 & $44 / 61$ & Australia & superficial; skin sore & [37] \\
\hline NS474 & 58 & Australia & superficial; wound infection & [37] \\
\hline NS488 & 12 & Australia & superficial; sinusitis; pharyngeal pus & {$[37]$} \\
\hline NS836 & $\mathrm{ck} 249$ & Australia & superficial; wound infection & [37] \\
\hline NS88.2rep & 98.1 & NA & isogenic $\operatorname{cov} S$ repaired NS88.2 strain & this study \\
\hline NS88.2covS & 98.1 & NA & reverse complemented NS88.2 covS mutant & this study \\
\hline
\end{tabular}

Clinical origin classified as invasive if infected tissue is normally sterile in a healthy host. STSS = Streptococcal toxic shock-like syndrome; $\mathrm{NF}=$ necrotising fasciitis; $\mathrm{NA}$ = not applicable.

Center, University of Tennessee Health Science Center by the use of MicroGrid II (Genomic Solutions). Additional 70mers, representing M1T1-specific prophages and prophage 3396 of $S$. $d y$ sgalactiae, were designed in batch according to the same design criteria applied for the other oligomers (Oligo Wiz 2.0, http:// www.cbs.dtu.dk/services/OligoWiz) and obtained from Integrated DNA Technologies.

\section{DNA-DNA Microarray}

DNA-DNA microarray experiments were conducted in dyeflipped biological triplicates for each GAS isolate, and all steps involving Alexa Fluor ${ }^{\circledR}$ dyes were conducted in the dark. RNAfree genomic DNA was extracted from overnight liquid cultures by a modified phenol-chloroform procedure [17] and randomly sheared into $<1-\mathrm{kb}$ fragments using a Misonix 3000 cup-horn sonicator and Branson Sonifier ${ }^{\circledR} 250$. Sheared DNA samples were fluorescently labeled with the BioPrime ${ }^{\circledR}$ Total Genomic Labeling
System (Invitrogen) as described by the manufacturer. In each hybridization reaction, equal amounts of Alexa Fluor ${ }^{\circledR}$ 3-labeled and Alexa Fluor ${ }^{\circledR}$ 5-labeled samples from different pairs of isolates were combined with hybridization buffer (Genisphere) and applied to the microarray slide. Following incubation at $55^{\circ} \mathrm{C}$ for $16 \mathrm{~h}$, glass slides were washed, dried via centrifugation and scanned using a GenePix 4000B scanner (Axon Instruments Inc.).

\section{DNA Microarray Data Analysis}

The GenePixPro 4.0 software (Axon Instruments Inc.) was used for primary analysis of the scanned GenePix files. The fluorescent intensities were then normalized to the median intensity for each channel. Data from all probes representing the same gene were averaged, and a mean hybridization score was calculated for each gene. An average threshold of 40 median-normalized fluorescence units was selected, under which a gene was called 'absent'. 


\section{Transcriptional Microarray}

Overnight GAS cultures were sub-inoculated 1:10 into fresh prewarmed media and grown to mid-logarithmic phase $\left(\mathrm{OD}_{600}\right.$ 0.4). Bacteria were concentrated 20-fold in Buffer RLT (Qiagen) containing $\beta$-mercaptoethanol, lysed by mechanical disruption in Lysing matrix B tubes (Q-Biogene) with a FastPrep FP120 Homogenizer (Q-Biogene) and flash frozen for storage at $-80^{\circ} \mathrm{C}$. Bacterial RNA was extracted using the RNeasy Mini Kit (Qiagen), treated with TURBO DNA-free ${ }^{\mathrm{TM}}$ DNase to remove contaminating genomic DNA (Ambion), re-concentrated on RNeasy columns (Qiagen) and converted to dendrimer-labeled cDNA with the Genisphere 3DNA Array 900MPX Kit as described by the corresponding manufacturer. Dendrimer-labeled cDNA samples from different pairs of isolates were combined and hybridized to the microarray slide for $16 \mathrm{~h}$ at $55^{\circ} \mathrm{C}$. Slides were washed to remove unbound $\mathrm{cDNA}$ and labeled with dendrimer-targeted Alexa Fluor 546 and Alexa Fluor 647 dyes for $5 \mathrm{~h}$ at $55^{\circ} \mathrm{C}$. Following a final wash to remove excess fluorescent dye, slides were coated in DyeSaver 2 (Genisphere) to preserve the fluorescent signal, and subsequently polished with toluene/acetone $(3: 1, \mathrm{v} / \mathrm{v})$ to minimize background fluorescence immediately prior to scanning in a GenePix 4000B scanner (Axon Instruments Inc.). Scanned images were processed with GenePixPro 4.0 software (Axon Instruments Inc.), and all transcriptional and statistical analyses undertaken in silico using GeneSpring GX 10 (Agilent Technologies). All transcriptional microarray data were submitted to the NCBI Gene Expression Omnibus (GEO) according to the MIAME standards (GEO accession No. GSE23825).

\section{Neutrophil Killing Assays}

The capacity of GAS isolates to survive during co-incubation with human neutrophils in vitro was determined as described by Hollands et al. [25]. Briefly, $2 \times 10^{4}$ colony-forming units (CFU) of mid-logarithmic-phase bacteria were incubated with $2 \times 10^{5}$ neutrophils in RPMI with $2 \%$ heat-inactivated plasma for $30 \mathrm{~min}$ at $37^{\circ} \mathrm{C}$. The final percent survival was calculated following comparison to the same bacterial culture incubated under the same conditions in the absence of neutrophils.

\section{In vivo Phase-Switching}

To examine the capacity for a phenotypic phase switch through $\operatorname{cov} R S$ mutation in vivo, sublethal doses of SpeB-positive GAS isolates, in the order of $10^{7} \mathrm{CFU}$ per dose, were subcutaneously administered in sterile $0.7 \%(\mathrm{w} / \mathrm{v}) \mathrm{NaCl}$ to the right flank of C57BL/ J6 mice less than 8 months of age (10 animals per isolate). On the third day after infection, mice were sacrificed by $\mathrm{CO}_{2}$ asphyxiation and the infected cutaneous lesions surgically removed. In vivo passaged bacteria were recovered from murine lesions on horse-blood agar and single colonies assayed for SpeB status as outlined above.

\section{Transgenic Murine Infection Model}

Humanized plasminogen transgenic ( $\mathrm{Tg}+$ ) AlbPLG1 mice, heterozygous for the human plasminogen gene [26], served as the animal model for determining GAS invasive potential as previously described [14]. GAS isolates were grown to logarithmic phase $\left(\mathrm{OD}_{600} 0.6\right)$, washed with sterile $0.7 \%(\mathrm{w} / \mathrm{v}) \mathrm{NaCl}$ and appropriately diluted to prepare the inoculum. Final dose of viable bacteria was confirmed using a plate-based serial dilution cultured on horse-blood agar. For each GAS isolate, a cohort of 10 humanized mice were subcutaneously challenged in the right flank and mortality was documented over a 10-day period.

\section{Restoration of covS in NS88.2}

The covS gene from NS88.2 was amplified using primers pHYcovSF (5'-gggggatccatggaaaatcagaaacaaaaacag-3') and pHYcovSR (5'-ggggaattcctaactctctttagactgggcc- $\left.3^{\prime}\right)$. The resulting amplicon was cloned into the temperature-sensitive vector pHY304 using BamHI/EcoRI restriction-enzyme digestion and ligation with T4 DNA ligase. Site-directed mutagenesis of the adenine nucleotide at position 581 to guanine was performed according to the method of Sanderson-Smith et al. [27], using

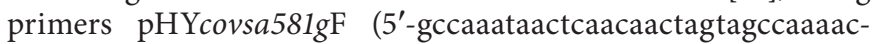

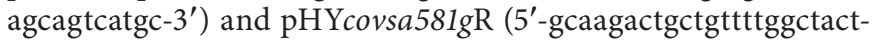
agttgttgagttatttggc- $3^{\prime}$ ). The resulting plasmids (pHYcovS and pHYrep) were transformed into Escherichia coli MC1061 using standard electroporation procedures. Allelic replacement mutants were constructed as described previously [28]. The covRS operons of the isogenic mutants NS88.2rep and NS88.2covS were sequenced as outlined above to confirm both the presence of the desired mutation and the integrity of the covRS operon.

\section{GAS Surface Plasmin Activity Assays}

GAS were incubated in human plasma as described previously [11]. Overnight growth in Todd-Hewitt broth supplemented with $1 \%(\mathrm{w} / \mathrm{v})$ yeast extract was diluted to $\mathrm{OD}_{600} 0.5$ and co-incubated with human plasma at $37^{\circ} \mathrm{C}$ for $3 \mathrm{~h}$. GAS were twice washed with PBS, $0.01 \%$ gelatin and $0.01 \mathrm{M}$ EDTA, prior to resuspension in PBS and $0.01 \%$ gelatin. Plasmin activity was determined using the chromogenic substrate Spectrozyme PL (American Diagnostica).

\section{Results}

\section{Expression of SpeB, Capsule Production and covRS \\ Mutation of Non-M1 GAS}

A range of GAS clinical isolates of differing emm types from invasive and benign infections were selected for this study (table 1). The well-characterized M1T1 clinical isolate 5448 and the natural isogenic covS mutant 5448AP [14] were included for comparison. With the exception of 3 strains, 5448AP, NS88.2 and A20, each of the GAS isolates described here expressed and secreted active SpeB at the stationary phase of growth (fig. 1a, b). An inverse correlation between $\mathrm{SpeB}$ expression and hyaluronic acid capsule production was observed, with 5448AP, NS88.2 and A20 hyperencapsulated with respect to all SpeB-positive GAS isolates (fig. 1c). GAS M1T1 strain 5448AP contains an adenine insertion at nucleotide position 887 in the ORF of the $\operatorname{cov} S$ gene, resulting in the premature truncation of the translated CovS protein [14]. Correspondingly, DNA sequence analysis of the SpeB-negative hyperencapsulated strains NS88.2 and A20 revealed a guanine to adenine substitution at position 581 in the 


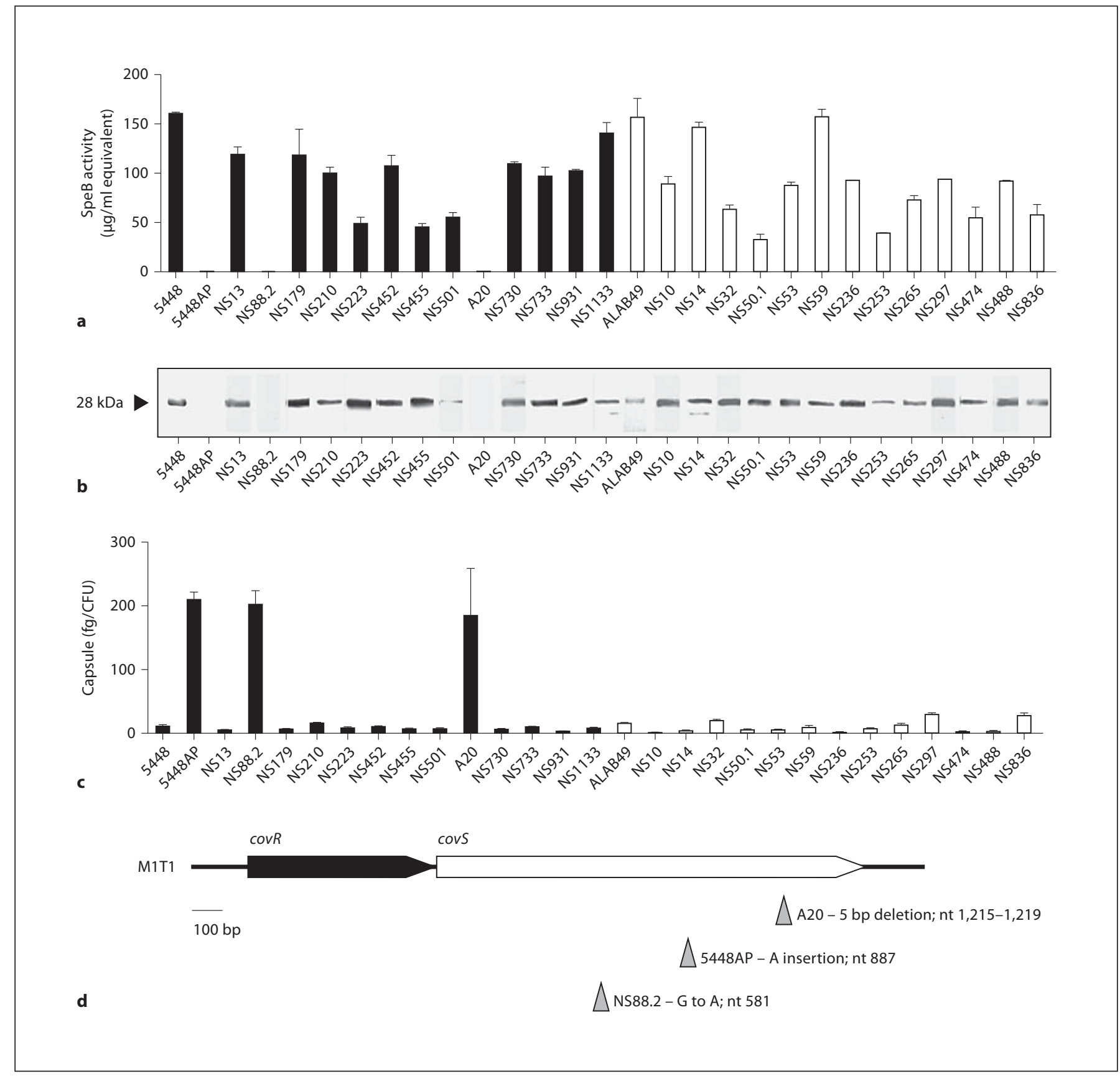

Fig. 1. Molecular and phenotypic analyses of GAS isolates representing distinct $M$ serotypes. a SpeB activity in cell-free stationary-phase supernatants of invasive (filled bars) and uncomplicated infection (open bars) GAS isolates. Isolate 5448 represents the globally disseminated M1T1 clone, while 5448AP is a hypervirulent animal passaged variant of 5448 [14]. b Western blot detection of SpeB in stationary-phase GAS supernatants. The $28-\mathrm{kDa}$ mature SpeB protease is indicated with an arrowhead. c Hyal- uronic acid capsule biomass of mid-logarithmic-phase invasive (filled bars) and uncomplicated infection (open bars) GAS isolates. d Schematic representation of the covRS operon. DNA sequence analysis confirmed the presence of inactivating covS mutations in the SpeB-deficient isolates A20, NS88.2 and 5448AP. The nature and nucleotide positions of the mutations in each isolate are indicated by the corresponding arrowheads. 
Fig. 2. In vitro mid-logarithmic phase transcriptional microarray analysis of $\operatorname{cov} R S$ intact and covRS mutant non-M1 GAS. a $\log _{10}$-fold differential expression of virulence-associated and regulatory genes of the covS mutants 5448AP (filled circles), A20 (open circles) and NS88.2 (shaded circles) compared to the covRS intact M1T1 strain 5448 . Selected genes are significantly differentially expressed in 5448AP with respect to 5448 ( $\mathrm{p}<0.05)$. b Principal component analysis on the non-M1 isolates ALAB49 (M53) and NS730 (M90), NS88.2 (M98.1) and A20 (M23), in addition to the M1T1 reference strains 5448 and 5448AP, revealed 2 distinct expression profiles in this 6-isolate strain set. Solid-line boxes placed in the plot area highlight strain clusters. The dashed-line box highlights the M1T1 strains 5448 and 5448AP, which, apart from a single base insertion in the 5448AP covS gene, harbor identical genomes.

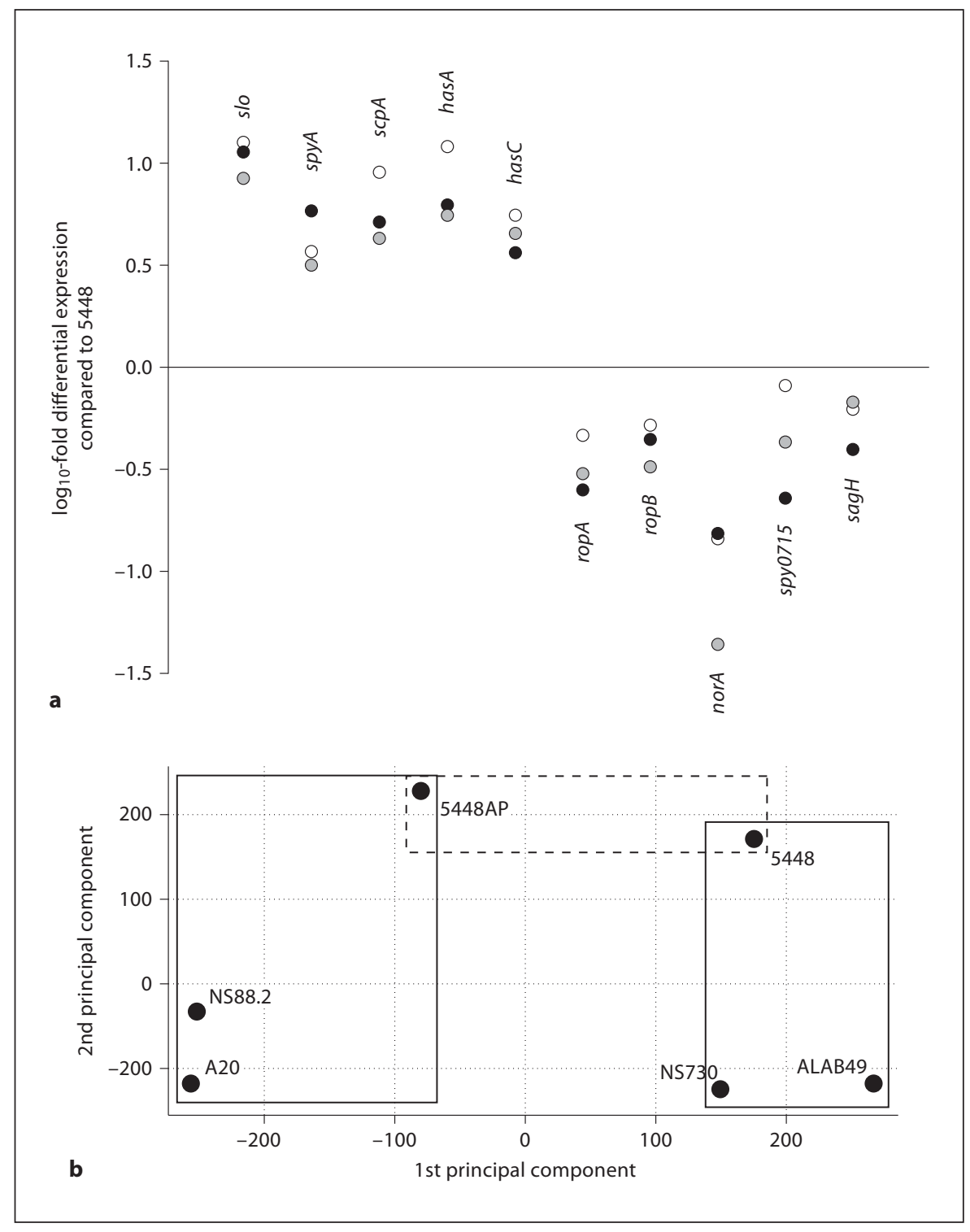

covS gene of NS88.2 and a 5-base pair deletion between positions 1,215 and 1,219 of the A20 covS gene (fig. 1d). Each mutation results in premature truncation of the translated CovS protein.

\section{DNA Microarray and Transcriptomic Analyses}

Three GAS strains with intact $\operatorname{cov} R S$ (5448, ALAB49 and NS730) and $3 \operatorname{covS}$ mutant forms (5448AP, NS88.2 and A20) were subjected to DNA microarray and transcriptomic analyses. DNA microarray identified 1,580 genes representing the core genome of the 6 GAS strains under examination (online suppl. table 1 and online suppl. fig. 1, www.karger.com/doi/10.1159/000317640).
The bacteriophage-encoded sdal gene, which confers on M1T1 GAS the capacity to switch to the $\operatorname{cov} R S$ mutant form at high frequency [14], was not present in the genomes of the non-M1 GAS strains (online suppl. table 1 and online suppl. fig. 1). Only ubiquitous genes were included in subsequent transcriptomic analyses undertaken on GAS strains grown to mid-logarithmic phase. The virulence-related genes found to be strongly upregulated in the $\operatorname{cov} S$ mutant strains, in comparison to the $\operatorname{cov} R S$ intact strain 5448, include genes of the has operon (has A, has $B$ and hasC; capsule biosynthesis), slo (streptolysin O) and the spyA exotoxin. Corroborating studies on M1T1 $\operatorname{cov} S$ mutation [13], the positive regulators of SpeB activ- 


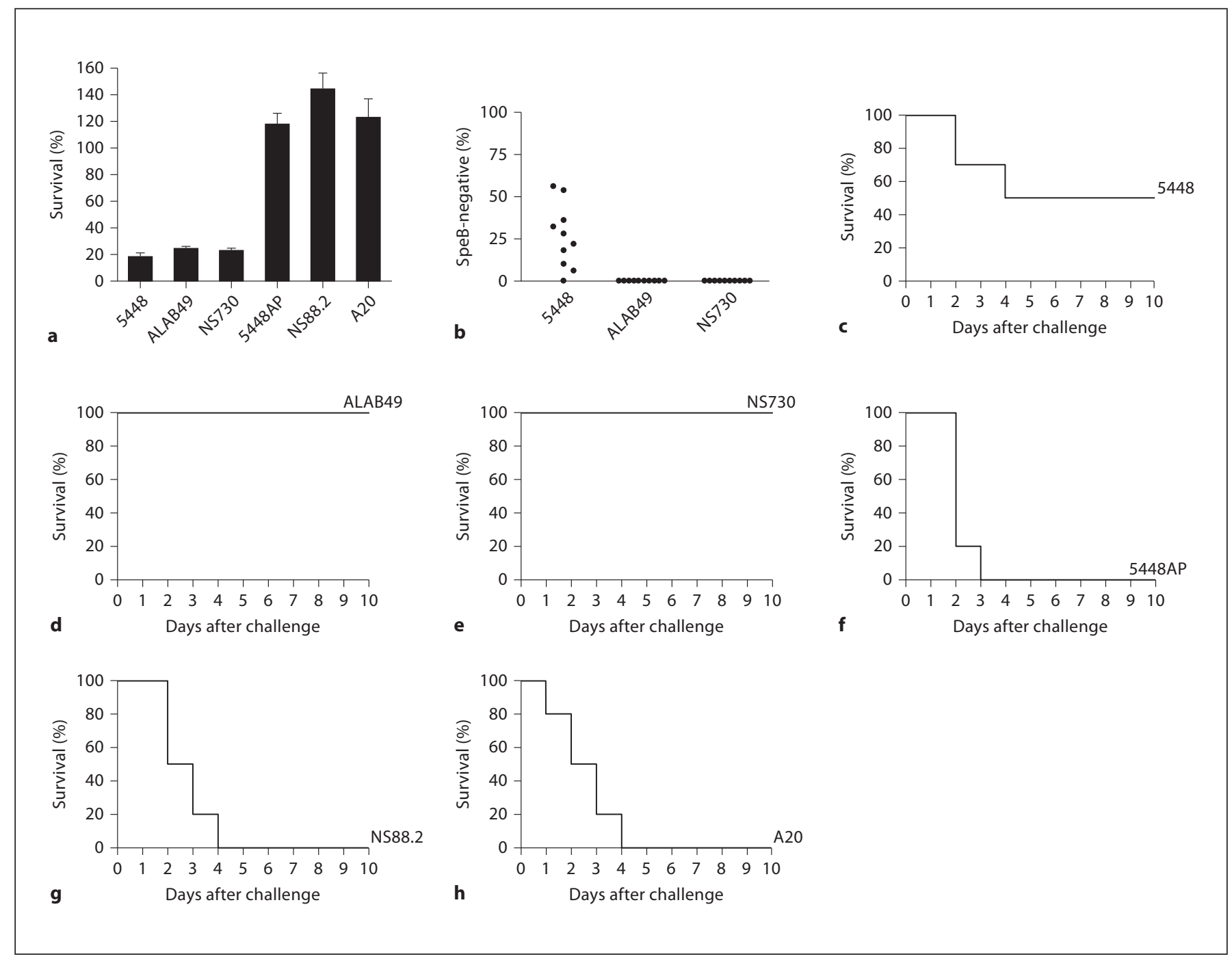

Fig. 3. Characterization of the $\operatorname{cov} R S$ intact isolates ALAB49 (M53) and NS730 (M90), and the covS mutant isolates NS88.2 (M98.1) and A20 (M23), in comparison with the M1T1 reference strains 5448 and 5448AP. a The percent survival of GAS isolates during co-culture with human neutrophils in vitro. $\mathbf{b}$ The capacity of SpeB-positive isolates to phase-switch to a SpeB-negative phenotype was assessed following a 3-day subcutaneous passage in C57BL/J6 mice. Each data point represents the percent of SpeBnegative $\operatorname{cov} R S$ mutants recovered from the infection site of each mouse ( $\mathrm{n}=10$ mice per strain). Subcutaneous infection of humanized plasminogen transgenic AlbPLG1 $(\mathrm{n}=10) 5448\left(3.9 \times 10^{7}\right.$ CFU/dose) (c), ALAB49 $\left(3.7 \times 10^{8}\right.$ CFU/dose $)(\mathbf{d}), \operatorname{NS} 730(2.2 \times$ $10^{8} \mathrm{CFU} /$ dose $)(\mathbf{e}), 5448 \mathrm{AP}\left(5.1 \times 10^{7} \mathrm{CFU} /\right.$ dose $)(\mathbf{f}), \mathrm{NS} 88.2(2.0$ $\times 10^{7} \mathrm{CFU} /$ dose $)(\mathbf{g})$ and A20 $\left(1.2 \times 10^{8} \mathrm{CFU} /\right.$ dose $)(\mathbf{h})$. ity and expression ropA and ropB $[29,30]$, in addition to genes of the $\operatorname{sag}$ operon $(\operatorname{sagB}, \operatorname{sagC}$ and $\operatorname{sagH})$, involved in streptolysin $S$ production [31], were found to be downregulated in the $\operatorname{cov} S$ mutant strains studied here (fig. 2a). No significant SpeB expression was detected at the midlogarithmic phase of growth, in accordance with previous studies [32]. Principal component analysis of these transcriptomic data revealed 2 distinct clusters of $\operatorname{cov} R S$ intact strains and $\operatorname{cov} S$ mutant forms (fig. 2b). These data suggest that differing $\mathrm{M}$ types harboring $\operatorname{cov} S$ mutations express related transcriptomic profiles.

\section{Neutrophil Resistance, SpeB Switching and Virulence}

In comparison with the covRS intact strains 5448, NS730 and ALAB49, the hyperencapsulated covS mutant strains 5448AP, NS88.2 and A20 displayed enhanced resistance to human neutrophil killing (fig. 3a). Of the covRS intact strains, the M1T1 strain 5448 readily 


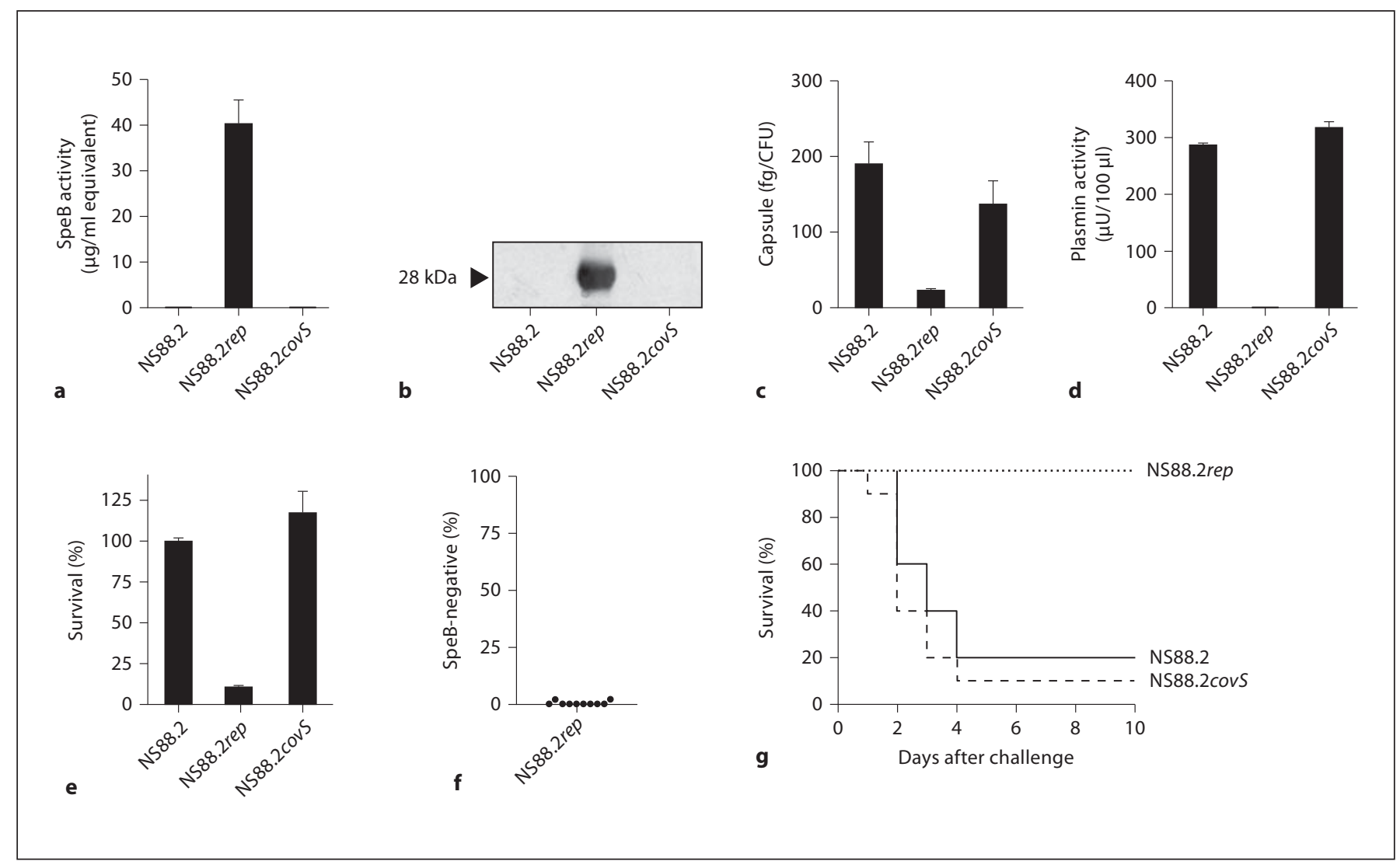

Fig. 4. Characterization of NS88.2 covS mutant (NS88.2 and NS88.2covS) and intact (NS88.2rep) isogenic strains. a SpeB activity in stationary-phase GAS supernatants. b Western blot detection of SpeB in stationary-phase supernatants. The mature SpeB protease is indicated with an arrowhead. c Hyaluronic acid capsule biomass of mid-logarithmic phase isogenic GAS strains. d Acquired surface plasmin activity following incubation in human plasma. e Percent survival following co-culture with hu-

switched to the SpeB-negative covRS mutant form in vivo (fig. 3b), in accordance with previous studies [11, 14, 20]. However, the $\operatorname{cov} R S$ intact non-M1 strains NS730 and ALAB 49 only infrequently switch to a covRS mutant form in vivo (fig. $3 b$ ). Utilizing the humanized plasminogen transgenic mouse line AlbPLG1, we assessed the virulence of both $\operatorname{cov} R S$ intact and mutant GAS strains. The M1T1 strain 5448, which has the capacity to switch at a high frequency to the covRS mutant form in vivo, was virulent in this mouse model (fig. 3c), corroborating previous work $[11,14]$. The covRS intact strains NS730 and ALAB49, which infrequently switch to the $\operatorname{cov} R S$ mutant form (fig. 3b), failed to establish a lethal infection (fig. 3d, e). Each of the $\operatorname{cov} S$ mutant strains, 5448AP, NS88.2 and A20, were highly virulent (fig. $3 \mathrm{f}-\mathrm{h}$ ). These data suggest man neutrophils in vitro. $\mathbf{f}$ The capacity of the SpeB-positive NS88.2rep to phase-switch to a SpeB-negative phenotype following a 3-day subcutaneous passage in C57BL/J6 mice. g Subcutaneous infection of humanised plasminogen transgenic AlbPLG1 mice $\left(\mathrm{n}=10\right.$ mice per strain) with NS88.2 $\left(1.0 \times 10^{7} \mathrm{CFU} /\right.$ dose $)$, NS88.2rep $\left(1.3 \times 10^{7} \mathrm{CFU} /\right.$ dose $)$ and NS88.2covS $\left(9.8 \times 10^{6} \mathrm{CFU} /\right.$ dose).

that while $\operatorname{cov} R S$ mutation may occur only infrequently in non-M1 GAS, such covRS mutant forms are hypervirulent.

\section{Repair of the covS Mutation in GAS Strain NS88.2 and Phenotypic Characterization}

In order to investigate whether low-frequency switching of non-M1 GAS results in the hypervirulent $\operatorname{cov} R S$ mutant form, the non-M1 GAS strain NS88.2 harboring a $\operatorname{cov} S$ mutation was chosen. The adenine nucleotide point mutation at position 581 in the NS88.2 $\operatorname{cov} S$ gene (fig. 1d) was converted to guanine by allelic replacement mutagenesis to construct strain NS88.2rep, with an intact or 'repaired' covS gene. Then, in order to fulfill Koch's molecular postulates [33], allelic replacement mutagene- 
sis was undertaken on NS88.2rep to restore the original adenine nucleotide point mutation, resulting in strain $\mathrm{NS} 88.2 \operatorname{cov} S$.

Repair of the covS mutation restored SpeB expression and activity in NS88.2rep, while NS88.2 and NS88.2covS remainedSpeB-negative (fig. 4a,b).NS88.2 and NS88.2covS were hyperencapsulated in comparison to NS88.2rep (fig. 4c), and acquired substantial surface plasmin activity following incubation in human plasma (fig. 4d). Both NS88.2 and NS88.2covS also displayed enhanced resistance to killing by human neutrophils (fig. 4e). The covS intact NS88.2rep displayed only limited capacity to switch to the more virulent covRS mutant form (fig. $4 \mathrm{f}$ ) and was not virulent in comparison to NS88.2 and NS88.2covS in AlbPLG1 mice (fig. 4g). These data support the hypothesis that non-M1 GAS serotypes switch less frequently to the hypervirulent $\operatorname{cov} R S$ mutant form, providing an explanation for the comparatively less frequent isolation of non-M1 serotypes from invasive human infections.

\section{Discussion}

Severe group A streptococcal invasive disease progresses rapidly and results in high patient morbidity, with approximately one quarter of cases being fatal despite the susceptibility of the pathogen to antibiotic treatment $[1$, 34]. Host genetic factors [35] and the human fibrinolytic protease plasmin $[26,36]$ have both been documented as contributing to GAS invasive disease potential. Whilst progression of GAS disease from benign mucosal infections to invasive disease occurs infrequently, the M1T1 clone is clinically and epidemiologically associated with deep tissue infections in Western countries [4]. Mutations in the M1T1 covRS locus result in a hyperencapsulated, SpeB-negative, hypervirulent phenotype, and are correlated with invasive diagnosis in patients $[10,12,13]$. The capacity of M1T1 to switch to the covRS mutant form at high frequency may result from acquisition of the phage borne sda 1 gene by M1T1 which confers resistance to neutrophil killing [14].

The contribution of similar mutations in $\operatorname{cov} R S$ to invasive disease potential of non-M1 GAS remains largely unknown. Many differing $M$ types have been associated with invasive infections $[21,34,37,38]$. A naturally occurring mutation in the $\operatorname{cov} R$ gene of an M3 isolate from a case of streptococcal toxic shock-like syndrome has been described. This $\operatorname{cov} R$ mutation was associated with increased capsule expression and enhanced virulence [39]. Mutation in $\operatorname{cov} R$, associated with enhanced expression of the interleukin- 8 cleaving protease SpyCEP, has also been observed in an M81 serotype GAS isolated from a lethal case of bacteremia and necrotizing fasciitis [40]. A correlation between mutation in global gene regulators $(\operatorname{cov} R S$ and $\operatorname{rop} B)$ and invasive pathology has been documented in a range of $\mathrm{M}$ types. In comparison to mutations in $r o p B$, inactivation of $\operatorname{cov} S$ is clinically predominant and results in greater virulence in murine infection models [21].

In this study, a variety of non-M1 GAS strains from invasive disease episodes were examined, including isolates representing the emm sequence types $14,22,23,25$, 52, 53, 69, 90, 91, 98.1 and 101. These included SpeB-positive isolates and SpeB-negative covS mutant forms. We propose that divergent $\mathrm{M}$ types with intact covRS possess an underlying capacity to cause invasive infection. In each serotype background, the covRS mutant form represents a more virulent state, which has greater propensity to cause invasive infection.

Using allelic replacement mutagenesis, we demonstrate that repair of the covS defect in the invasive emm 98.1 GAS strain NS88.2 (covS mutant form) renders the isogenic covS intact strain NS88.2rep SpeB-positive, susceptible to neutrophil killing and less able to accumulate surface plasmin activity following growth in human plasma. The covS intact NS88.2rep strain was also found to be avirulent in the humanized plasminogen transgenic mouse model. In comparison to the M1T1 GAS covS intact strain 5448, we propose that the lack of virulence of NS88.2rep is due to the reduced capacity of this strain to switch to the invasive covRS mutant form in vivo. The lower frequency of switching to the $\operatorname{cov} S$ mutant form limits the number of these invasive variants at the site of local infection. This lower frequency of switching may reflect the absence of the sdal gene in this genetic background. The reduced capacity of non-M1 GAS serotypes to switch to the hypervirulent $\operatorname{cov} R S$ mutant form may provide an explanation for the comparatively less frequent isolation of non-M1 serotypes from invasive human infections.

\section{Acknowledgments}

P.M. and A.H. are the recipients of Australian Postgraduate Awards. J.C. is the recipient of an Australian National Health and Medical Research Council Overseas Biomedical Training Fellowship (514639). This work was funded by NIH grant AI077780 (V.N., M.W.), National Health and Medical Research Council of Australia Grant 573401 (M.W.) and an Australian International Science Linkages grant CG110095 (M.W., M.K., V.N., A.H.). 


\section{References}

-1 Carapetis JR, Steer AC, Mulholland EK, Weber M: The global burden of group A streptococcal diseases. Lancet Infect Dis 2005;5: 685-694.

-2 Tart AH, Walker MJ, Musser JM: New understanding of the group A Streptococcus pathogenesis cycle. Trends Microbiol 2007; 15:318-325

3 Musser JM, Kapur V, Szeto J, Pan X, Swanson DS, Martin DR: Genetic diversity and relationships among Streptococcus pyogenes strains expressing serotype M1 protein - recent intercontinental spread of a subclone causing episodes of invasive disease. Infect Immun 1995;63:994-1003.

4 Aziz RK, Kotb M: Rise and persistence of global M1T1 clone of Streptococcus pyogenes. Emerg Infect Dis 2008;14:1511-1517.

$\checkmark 5$ Hassell M, Fagan P, Carson P, Currie BJ: Streptococcal necrotising fasciitis from diverse strains of Streptococcus pyogenes in tropical northern Australia: case series and comparison with the literature. BMC Infect Dis 2004; $4: 60$.

6 Ravins M, Jaffe J, Hanski E, Shetzigovski I, Natanson-Yaron S, Moses AE: Characterization of a mouse-passaged, highly encapsulated variant of group A Streptococcus in in vitro and in vivo studies. J Infect Dis 2000; 182:1702-1711.

$\checkmark 7$ Moses AE, Wessels MR, Zalcman K, Alberti S, Natanson-Yaron S, Menes T, Hanski E: Relative contributions of hyaluronic acid capsule and $\mathrm{M}$ protein to virulence in a mucoid strain of the group A Streptococcus. Infect Immun 1997;65:64-71.

$>8$ Johnson DR, Stevens DL, Kaplan EL: Epidemiologic analysis of group A streptococcal serotypes associated with severe systemic infections, rheumatic fever, or uncomplicated pharyngitis. J Infect Dis 1992;166:374-382.

-9 Marcon MJ, Hribar MM, Hosier DM, Powell DA, Brady MT, Hamoudi AC, Kaplan EL: Occurrence of mucoid M-18 Streptococcus pyogenes in a central Ohio pediatric population. J Clin Microbiol 1988;26:1539-1542.

>10 Engleberg NC, Heath A, Miller A, Rivera C, DiRita VJ: Spontaneous mutations in the CsrRS two-component regulatory system of Streptococcus pyogenes result in enhanced virulence in a murine model of skin and soft tissue infection. J Infect Dis 2001;183:10431054.

- 11 Cole JN, McArthur JD, McKay FC, Sanderson-Smith ML, Cork AJ, Ranson M, Rohde M, Itzek A, Sun H, Ginsburg D, Kotb M, Nizet V, Chhatwal GS, Walker MJ: Trigger for group A streptococcal M1T1 invasive disease. FASEB J 2006;20:1745-1747.
12 Kansal RG, McGreer A, Low DE, NorrbyTeglund A, Kotb M: Inverse relation between disease severity and expression of the streptococcal cysteine protease, SpeB, among clonal M1T1 isolates recovered from invasive group A streptococcal infection cases. Infect Immun 2000;68:6362-6369.

13 Sumby P, Whitney AR, Graviss EA, DeLeo FR, Musser JM: Genome-wide analysis of group A streptococci reveals a mutation that modulates global phenotype and disease specificity. PLoS Pathog 2006;2:e5.

14 Walker MJ, Hollands A, Sanderson-Smith M, Cole JN, Kirk JK, Henningham A, McArthur JD, Dinkla K, Aziz RK, Kansal RG, Simpson AJ, Buchanan JT, Chhatwal GS Kotb M, Nizet V: DNase Sda1 provides selection pressure for a genetic and phenotypic switch promoting invasive group A streptococcal infection. Nat Med 2007;13:981-985.

15 Levin JC, Wessels MR: Identification of $\operatorname{csrR} / \operatorname{csr} S$, a genetic locus that regulates hyaluronic acid capsule synthesis in group A Streptococcus. Mol Microbiol 1998;30:209219.

16 Sumby P, Porcella SF, Madrigal AG, Barbian KD, Virtaneva K, Ricklefs SM, Sturdevant DE, Graham MR, Vuopio-Varkila J, Hoe NP, Musser JM: Evolutionary origin and emergence of a highly successful clone of serotype M1 group A Streptococcus involved multiple horizontal gene transfer events. J Infect Dis 2005;192:771-782.

17 Aziz RK, Edwards RA, Taylor WW, Low DE, McGreer A, Kotb M: Mosaic prophages with horizontally acquired genes account for the emergence and diversification of the globally disseminated M1T1 clone of Streptococcus pyogenes. J Bacteriol 2005; 187:3311-3318.

18 Sumby P, Barbian KD, Gardner DJ, Whitney AR, Welty DM, Long RD, Bailey JR, Parnell MJ, Hoe NP, Adams GG, Deleo FR, Musser JM: Extracellular deoxyribonuclease made by group A Streptococcus assists pathogenesis by enhancing evasion of the innate immune response. Proc Natl Acad Sci USA 2005;102:1679-1684.

19 Buchanan JT, Simpson A, Aziz R, Liu G, Kristian S, Kotb M, Feramisco J, Nizet V: DNase expression allows the pathogen group A Streptococcus to escape killing in neutrophil extracellular traps. Curr Biol 2006;16: 396-400.

20 Aziz RK, Pabst MJ, Jeng A, Kansal R, Low DE, Nizet V, Kotb M: Invasive M1T1 group A Streptococcus undergoes a phase-shift in vivo to prevent proteolytic degradation of multiple virulence factors by SpeB. Mol Microbiol 2004;51:123-134.

-21 Ikebe T, Ato M, Matsumura T, Hasegawa H, Sata T, Kobayashi K, Watanabe H: Highly frequent mutations in negative regulators of multiple virulence genes in group A streptococcal toxic shock syndrome isolates. PLoS Pathog 2010;6:e1000832.
22 Hytönen J, Haataja S, Gerlach D, Podbielski A, Finne J: The SpeB virulence factor of Streptococcus pyogenes, a multifunctional secreted and cell surface molecule with strepadhesin, laminin-binding and cysteine protease activity. Mol Microbiol 2001;39: 512-519.

23 Ashbaugh CD, Warren HB, Carey VJ, Wessels MR: Molecular analysis of the role of the group A streptococcal cysteine protease, hyaluronic acid capsule, and $\mathrm{M}$ protein in a murine model of human invasive soft-tissue infection. J Clin Invest 1998;102:550-560.

24 Ashbaugh CD, Wessels MR: Absence of a cysteine protease effect on bacterial virulence in two murine models of human invasive group A streptococcal infection. Infect Immun 2001;69:6683-6688.

25 Hollands A, Aziz RK, Kansal RG, Kotb M, Nizet V, Walker MJ: A naturally occuring mutation in ropB suppresses SpeB expression and reduces M1T1 group A streptococcal systemic virulence. PLoS One 2008;3:e4102.

26 Sun H, Ringdahl U, Homeister JW, Fay WP, Engleberg NC, Yang AY, Rozek LS, Wang X, Sjöbring U, Ginsburg D: Plasminogen is a critical host pathogenicity factor for group A streptococcal infection. Science 2004;305: 1283-1286

27 Sanderson-Smith ML, Walker MJ, Ranson M: The maintenance of high affinity plasminogen binding by group A streptococcal plasminogen-binding M-like protein is mediated by arginine and histidine residues within the a1 and a2 repeat domains. J Biol Chem 2006;281:25965-25971.

28 Sanderson-Smith ML, Dinkla K, Cole JN, Cork AJ, Maamary PG, McArthur JD, Chhatwal GS, Walker MJ: M protein-mediated plasminogen binding is essential for the virulence of an invasive Streptococcus pyogenes isolate. FASEB J 2008;22:2715-2722.

29 Lyon WR, Gibson CM, Caparon MG: A role for Trigger Factor and an Rgg-like regulator in the transcription, secretion and processing of the cysteine proteinase of Streptococcus pyogenes. EMBO J 1998;17:6263-6275.

30 Neely MN, Lyon WR, Runft DL, Caparon M: Role of RopB in growth phase expression of the SpeB cysteine protease of Streptococcus pyogenes. J Bacteriol 2003;185:5166-5174.

-31 Nizet V, Beall B, Bast DJ, Datta V, Kilburn L, Low DE, De Azavedo JCS: Genetic locus for streptolysin S production by group A Streptococcus. Infect Immun 2000;68:4245-4254.

-32 Unnikrishnan M, Cohen J, Sriskandan S: Growth-phase-dependent expression of virulence factors in an M1T1 clinical isolate of Streptococcus pyogenes. Infect Immun 1999; 67:5495-5499.

>33 Falkow S: Molecular Koch's postulates applied to microbial pathogenicity. Rev Infect Dis 1988;10:S274-S276. 
34 Cunningham MW: Pathogenesis of group A streptococcal infections. Clin Microbiol Rev 2000;13:470-511.

-35 Kotb M, Norrby-Teglund A, McGeer A, ElSherbini H, Dorak MT, Khurshid A, Green K, Peeples J, Wade J, Thomson G, Schwartz B, Low DE: An immunogenetic and molecular basis for differences in outcomes of invasive group A streptococcal infections. Nat Med 2002;8:1398-1404.

-36 Walker MJ, McArthur JD, McKay F, Ranson M: Is plasminogen deployed as a Streptococcus pyogenes virulence factor? Trends Microbiol 2005;13:308-313.
37 McKay FC, McArthur JD, Sanderson-Smith ML, Gardam S, Currie BJ, Sriprakash KS, Fagan PK, Towers RJ, Batzloff MR, Chhatwal GS, Ranson M, Walker MJ: Plasminogen binding by group A streptococcal isolates from a region of hyperendemicity for streptococcal skin infection and a high incidence of invasive infection. Infect Immun 2004;72: 364-370.

-38 Fiorentino TR, Beall B, Mshar P, Bessen DE: A genetic-based evaluation of the principal tissue reservoir for group A streptococci isolated from normally sterile sites. J Infect Dis 1997; 176:177-182.

-39 Miyoshi-Akiyama T, Ikebe T, Watanabe H, Uchiyama T, Kirikae T, Kawamura Y: Use of DNA arrays to identify a mutation in the negative regulator, $\operatorname{cs} r$, responsible for the high virulence of a naturally occurring type M3 group A Streptococcus clinical isolate. J Infect Dis 2006;193:1677-1684.
40 Turner CE, Kurupati P, Jones MD, Edwards RJ, Sriskandan S: Emerging role of the interleukin-8 cleaving enzyme SpyCEP in clinical Streptococcus pyogenes infection. J Infect Dis 2009;200:555-563.

41 Okamoto H, Shoin S, Minami M, Koshimur S, Shimizu R: Experimental anticancer studies 30. Factors influencing streptolysin Sforming ability of streptococci having anticancer activity. Jpn J Exp Med 1966;36:161.

42 Svensson MD, Sjöbring U, Luo F, Bessen DE: Roles of the plasminogen activator streptokinase and the plasminogen-associated M protein in an experimental model for streptococcal impetigo. Microbiol 2002;148: 3933-3945. 